LAS FUNCIONES

DEL PSICÓLOGO

EN UNA INSTITUCIÓN

DE EDUCACIÓN

ESPECIAL PRIVADA,

SEGÚN

\section{LAS PERCEPCIONES}

DE LOS DOCENTES

Marjorie Barquero

Ramírez*

\section{RESUMEN}

El presente estudio es una exploración descriptiva acerca de la percepción que del "rol del psicólogo educativo" tienen los docentes de una Escuela de Educación Especial. Dicho estudio se realizó en el Instituto Andrea Jiménez. Los resultados indican una percepción positiva hacia la inclusión del psicólogo educativo en el equipo de trabajo escolar y sugieren divulgación y capacitación acerca del rol del psicólogo educativo.

* Magíster en Psicología Educativa y trabaja como psicóloga en la Fundación Andrea Jiménez y en el Instituto Andrea Jiménez.

\section{INTRODUCCIÓN}

Los psicólogos costarricenses hemos sido testigos (y actores, en algunos casos) en las últimas dos décadas de la cada día más evidente "diferenciación" de la Psicología Educativa de otras ramas de la misma disciplina. Esta diferenciación no se limita a la inserción laboral: ser psicólogo educativo es más que ser un psicólogo que trabaja en una escuela, ó que trabaja con población en edad escolar... Thomas L. Good y Jere Brophy concluyeron, en 1999, al igual que lo hace la National Association of School Psychologists (NASP) con anterioridad, que el psicólogo educativo es el llamado a "proporcionar un marco de referencia para observar al estudiante, al proceso de aprendizaje y a la situación de aprendizaje", y que es en este intento que deja al descubierto su amplia formación y dominio del currículum escolar, del diseño institucional, del desarrollo-personalidadaptitud de los estudiantes, del proceso y métodos de aprendizaje (teorías de aprendizaje y motivación), de la dinámica social (organización y cognición social), del manejo e intervención conductual y de la evaluación constante de los resultados. Es decir que, da la "leve impresión" de ser algo más que "desarrollar suposiciones explícitas acerca de las condiciones que facilitan el aprendizaje de los estudiantes"... luce más como una intervención macro-psi- 
cosocial, enfocada en el rendimiento académico, pero que surge de la comprensión clínica tácita de la salud mental de todos los actores sociales implicados en el ambiente y proceso educativo. Okland T. \& Cunningham J. efectuaron, en 1996, una revisión histórica de la evolución de la Psicología Educativa en Norteamérica, señalando la existencia de modelos consecutivos para la comprensión de tal evolución y sus alcances futuros (abordaje lineal, abordaje analítico o de estadios, abordaje de contexto, y el modelo dialéctico), siendo lo cierto que la "diferenciación" de la psicología educativa se hace también allá evidente conforme nos acercamos al siglo XXI. El camino de tal diferenciación también se intuye desde ahí rico en complejidad, partiendo del reconocimiento de la Psicología como una ciencia y de la Psicología Educativa como una "diversificación" que la fortalece en la "especialización" misma.

Al igual que los colegas de los países más avanzados, ya los psicólogos costarricenses también llegamos a la necesidad de reconocer esta especialidad con la importancia social que conlleva, y por ende a fortalecerla.... Así pareciera imposible evitar la inminente revisión de nuestro Código de Ética, para con la inclusión de incisos específicos al ámbito y praxis "educativas", y ofrecer a esta especialidad el soporte gremial que le permitirá consolidarse y crecer con mayor rapidez, tal y como ya lo han hecho la APA y la OATA, entre otras. Es esa seguridad la que queda implícita en el "Manual de Conducta Profesional" de la NASP, donde se define al Psicólogo Educativo como aquel profesional en psicología que utiliza un proceso de toma de decisiones en equipo basado en consideraciones teóricas abundantes, que tiene la habilidad para escuchar, que trabaja con individuos aislados, grupos y sistemas, que desarrolla retos académicos y cognitivos para el alumnado, y provee información acerca de cómo los estudiantes logran esas metas y monitorea éstos procesos, que tiene la sensibilidad, conocimiento, y destreza para abordar sujetos de diferentes razas, condiciones económicas, culturas, experiencia y recursos lingüísticos, y que demuestra su conocimiento de programas escolares, proyectos, política y filosofía institucionales en función de la población beneficiaria; sin dejar de lado el hecho de que utiliza métodos preventivos, de promoción de la salud mental e intervención en crisis basado en su conocimiento del desarrollo del niño, la psicopatología, la diversidad y los estresores sociales, el cambio y los sistemas. Además, menciona la NASP, que el Psicólogo Educativo tiene conocimientos de las influen- 
cias familiares que afectan el bienestar de los alumnos, su aprendizaje y rendimiento, y se involucra en las políticas institucionales que promueven la interrelación entre padres, maestros y la comunidad. Es decir, el Servicio que ofrece el Psicólogo Educativo cubre funciones básicas de consultoría, asesoría, intervención psicológica (individual, grupal y de sistemas), prevención, manejo directo del alumno y su proceso de aprendizaje especial, e investigación y planeamiento (dentro y fuera del ambiente escolar), tal y como se desprende de la investigación teórica realizada para este estudio. En síntesis, se basa en el conocimiento esencial relativo a los fundamentos de la psicología, fundamentos de educación, la intervención y solución de problemas, la estadística e investigación metodológica, y la formación profesional como "Psicólogo Educativo" primordialmente (1994, Apendix III, Best Practices in School Psychology III). Claro está que este conocimiento debiera ofrecerse bajo el amparo de los valores éticos específicos y con la obvia articulación de filosofía, metas y objetivos de entrenamiento para la praxis futura. El desempeño de tan variada función implica, además, un rango amplísimo de ambientes de intervención (rural a urbano), que podrían "enmascarar" su verdadera y compleja función como "Psicólogo Educativo", haciéndonos pensar erróneamente que basta con tener capacitación en psicología para desempeñarse como "psicólogo" de una escuela. Así es como vemos hoy a profesionales en esta especialidad trabajando en clínicas, en agencias de adopciones o de organismos de caridad, en cortes juveniles, colegios, universidades, escuelas de educación especial, y hasta en escenarios de características médicas o servicios de especialidades médicas definidas. Con ello, la restringida y severa división entre Psicología Clínica y Psicología Educativa se desdibuja exigiendo a los actores profesionales de la segunda una formación en clínica y en psicología educativa para poder desempeñarse como psicólogo en cualquier ambiente del sistema educativo nacional, donde prioritariamente los niños-estudiantes serán sus clientes, pero donde necesariamente ha de lidiar con los asuntos de padres y maestros y hasta del sistema (escolar y social).

Tal y como lo menciona John Brown, en su artículo no publicado "Principios éticos y prácticos para el éxito en el desempeño del Psicólogo Educativo" (UNIBE, 2001), y coincidente con lo anotado por Sprinthall \& Sprinthall (1990), es precisamente la naturaleza híbrida de ciencia y educación, implícita en las funciones del Psicólogo Educativo lo que dificulta su comprensión, reflejándose ésto en su agenda cotidiana de 
cuatro vías inseparables de intervención: alumnos, docentes, familiares, y tareas administrativas.

El propósito de este estudio es conducir una exploración inicial del "Rol del Psicólogo Educativo" partiendo para ello de la percepción que los docentes de una escuela de Enseñanza Especial tengan en relación con: 1) la justificación de su inclusión en el equipo de trabajo escolar, 2) sus funciones y tareas específicas, 3) el horario de atención necesario, 4) el alcance del servicio sobre la población beneficiaria.; y 5) el perfil del psicólogo educativo en ese ambiente escolar.

La exploración se realiza con la totalidad de los docentes y asistentes con formación docente formal del Instituto Andrea Jiménez, escuela de Educación Especial que, desde su nacimiento, ha incluido en su equipo de trabajo a los Psicólogos, de una u otra manera. Al utilizar el presente estudio docentes de educación especial costarricenses, se accesa directamente a los recursos de formación formal y valores propios de la idiosincrasia tica, por lo que este estudio también podría reflejar, como tamizaje, la situación global del psicólogo educativo ya inserto en el ámbito y función específica.

\section{Metodología}

Participantes

El total de las docentes del Instituto Andrea Jiménez, escuela privada de educación especial que atiende población con discapacidad cognitiva, participó voluntariamente en este estudio. De las 22 docentes participantes, fue posible recopilar la información de 20 de ellas (90,9\%), pues hubo una devolución en blanco y otra no devolvió el instrumento.

En este grupo de docentes, cuatro se desempeñan como "aspirantes" $(20 \%)$, asistiendo en funciones docentes a las maestras de grupo; dos son maestras de materias específicas $(10 \%)$ como son Educación Física y Artes Plásticas; y de las profesoras tituladas, dos están calificadas ante el MEP como ET-2 (10\%), siete como ET-3 (35\%) y las cinco restantes como ET-5 (25\%).

A las maestras se les solicitó personalmente, y por escrito, que completaran de manera individual la información incluida en el instrumento "Escala de Percepción del Docente de Educación Especial en relación con el Rol del Psicólogo Educativo". La garantía de confidencialidad y anonimato para el personal participante se hizo efectiva con el procesamiento ciego de los datos, razón por la cual se les solicitó también que entregaran la escala dentro del sobre original sellado. 
El grupo de docentes fue invitado a una reunión en la cual recibieron devolución de los resultados de este estudio y se analizaron las recomendaciones derivadas de los mismos.

\section{Instrumentos}

Basado en la información recogida en múltiples fuentes publicadas (o aún inéditas) especialmente en las últimas dos décadas se construyó una escala de 30 ítemes específicamente para recopilar la percepción que del rol del "psicólogo educativo" tienen los docentes de Educación Especial ("Escala de Percepción del docente de Educación Especial en relación con el Rol del Psicólogo Educativo"). La escala es básicamente binaria, aún cuando cuenta con ampliaciones cualitativas, para precisar en la "percepción" de los participantes y lograr entonces "descripciones" fieles de lo arrojado por el procesamiento (ciego) de los datos recolectados. Cinco son los factores medidos por la escala: 1) el psicólogo como funcionario escolar, 2) identificación de tareas o funciones del "rol" del psicólogo, 3) horario del servicio de psicología, 4) alcances del servicio de psicología en el ambiente escolar, 5) perfil del psicólogo educativo.

En el factor 1 se incluye el reconocimiento del psicólogo como funcio- nario, el saberlo parte del equipo interdisciplinario con que cuenta la escuela, la identificación del psicólogo educativo dentro de la escuela de educación especial como un psicólogo con desempeño "particular". El factor 2 incluye la identificación de las tareas cotidianas en el desempeño actual del psicólogo escolar por área de atención, la definición del "rol ideal" del psicólogo educativo en el ambiente de la escuela de educación especial, tanto por área de atención específica como en el ambiente escolar general de acuerdo con los ámbitos tradicionales demarcadas por la "praxis psicológica". Por otra parte, el factor 3 pretende establecer el horario ideal de acuerdo con la cobertura en tiempo que implica la atención de las áreas de intervención pre-establecidas en la escala.

El factor 4 permite definir la percepción que el docente tiene del alcance del servicio ofrecido por el psicólogo educativo, en términos de su percepción relativa a la eficacia, funcionalidad, y accesibilidad de tal servicio en el desempeño de su encargo pedagógico, tanto con alumnos específicos, como con los programas pedagógicos y el proyecto institucional. Finalmente, el factor 5 permite detectar la percepción de las características personales y profesionales que ha de presentar el psicólogo educativo de la escuela 
de Enseñanza Especial desde la perspectiva del docente, como reflejo (o resonancia) de su percepción general del "rol" de éste.

Antes de su administración, la "validez" de esta escala fue consultada al solicitar su revisión por dos "psicólogos experimentados en el campo de la psicología educativa", luego de haber sido revisada y comentada por profesionales de otras áreas con la intención de confirmar la precisión en la "comprensión" original de cada ítem.

\section{Procedimiento}

Cada participante individual fue solicitado verbalmente a colaborar de manera voluntaria con el estudio, sirviéndose completar la información solicitada en la "escala de percepción". Ante el consentimiento verbal y voluntario, cada participante recibió dentro de un sobre opaco una copia de la "Escala de Percepción del docente de Educación Especial en relación con el Rol del Psicólogo Educativo", así como una nota de la investigadora donde explicaba el procesamiento ciego de los datos arrojados por el estudio como un garante de "confidencialidad y anonimato" para los participantes, así como un manejo profesional y ético de los resultados y sus inferencias. Esto, pues la investigadora está vinculada laboralmente a esa escuela. Además ahí se hace mención de la participación de dos colegas con experiencia en el campo educativo, quienes a su vez respaldan y garantizan tal procesamiento. Se indica a los participantes que, al completar la escala, la entreguen dentro del sobre original cerrado, directamente a la investigadora para evitar pérdida de datos. Se hizo siempre hincapié en que la escala se constituía en un instrumento individual, que no requería de comentarios gremiales o asociados, pero que en caso de requerir aclaración sobre algún ítem, la investigadora se mantendría a disposición de los participantes durante el tiempo que les tomara completar el instrumento (30-40 minuto[OB1]s aprox.).

\section{Análisis de los datos}

Los datos fueron recolectados por la investigadora a través de la aplicación individual y secreta de la escala de percepción construída para cubrir los objetivos pretendidos con este estudio. El procesamiento de la información fue ciega, trabajándose sólo sobre los porcentajes y totales en el procesamiento de estadística descriptiva e inferencial.

\section{Resultados}

De acuerdo con los factores intervinientes en la percepción que del 
"Rol del Psicólogo Educativo" tienen los docentes de Educación Especial de esta escuela, se concluye que:

- Percepción del Psicólogo como miembro del equipo de trabajo escolar:

Se parte de que todos los participantes conocieron $(100 \%)$ la existencia del "psicólogo" como miembro del equipo de trabajo escolar, señalando en ese mismo porcentaje que su inclusión es importante, necesaria y primordial para el funcionamiento del equipo interdisciplinario y la toma de decisiones asertiva en beneficio de la población atendida. Se percibe como un funcionario accesible, con buena disposición y capacitado.

- Percepción de las tareas y funciones del Psicólogo Educativo: El 95\% de las docentes participantes indicaron conocer las funciones del psicólogo de esta escuela, pese a que a la hora de indicar estas funciones sobre la lista de las funciones reales la identificación que se hace de éstas deja entrever el desconocimiento del detalle del desempeño cotidiano del psicólogo en su rol institucional:

- $60 \%$ menciona funciones con los alumnos;
- $65 \%$ menciona funciones con los docentes;

- $70 \%$ menciona funciones con los familiares;

- $75 \%$ menciona funciones administrativas.

A la hora de identificar las funciones ideales los porcentajes obtenidos son:

- con alumnos $80 \%$

- con docentes $70 \%$

- con familiares $85 \%$ administrativos $55 \%$

Es particularmente llamativa la referencia que se hace de la ejecución de funciones que no se consideran propias a la función, mencionándose como tales la asistencia y sustitución de la dirección, lectura de tesis, selección y entrenamiento de personal, y la organización de actividades institucionales. Lo anterior revela lo impreciso del papel del psicólogo educativo, tanto como la falta de información y capacitación al respecto. Especialmente desinformadas aparecen las "asistentes", cuya relación con el psicólogo está mediada por la docente de grupo.

La percepción de las funciones y tareas del psicólogo es difusa y poco precisa, aunque sí se detecta como un rol de funciones múltiples. 
- Percepción del horario del Servicio de Psicología en la escuela:

Parece existir una gran confusión y desconocimiento (65\%) en torno al horario del Psicólogo Educativo, considerándosele flexible y dispuesto, pero impreciso en sus horas de permanencia en la escuela. El horario ideal señalado para el buen desempeño de estas funciones es el tiempo completo $(70 \%)$, entendido éste por el tiempo de permanencia del alumnado en la escuela.

Se percibe la necesidad de contar con la cobertura del psicólogo en el horario de desarrollo del programa pedagógico, como fundamental.

- Percepción del alcance del Servicio de Psicología en el ambiente escolar (beneficiarios):

Alrededor del 50\% indican que el Servicio de psicología brinda en la actualidad la atención y cobertura que la población beneficiaria requiere (alumnos, docentes, familiares), justificando su posición en la acentuada ejecución de tareas administrativas adicionales que se le asignan durante el proceso. Pese a esto, todas las docentes participantes se manifiestan en la mayor disposición de mantenerse trabajando con el psicólogo educativo de la institución, pues contradictoriamente a lo anterior ahora se considera que éste brinda la información y el apoyo que como docentes requieren sobre sus alumnos $(90 \%)$, y el $100 \%$ reconoce que éste se encuentra familiarizado con el trabajo que se realiza en la escuela y en los diversos programas.

- En idéntico porcentaje reconocen haber trabajado con otros psicólogos, pero sólo el $25 \%$ de esos, reconocen el papel del psicólogo como diferente al de los psicólogos de otras instituciones.

- Percepción del Perfil del Psicólogo Educativo:

El 70\% de las participantes reconocen el papel del psicólogo de la institución como educativo, $65 \%$ como asesor, $60 \%$ como desempeño de una función psicosocial, y $45 \%$ lo señalan como clínico y consultor, $35 \%$ laboral, y sólo el 10\% de ellas tienen la claridad de que la función del Psicólogo Educativo involucra un desempeño múltiple.

De acuerdo con la formacióncapacitación establecida como parte del perfil que del psicólogo educativo da la NASP, las docentes logran mencionar actividades relativas exclusivamente a los fundamentos psico- 
lógicos y educativos, utilizando vocablos imprecisos o inespecíficos que pierden la riqueza de la información. Situación similar ocurre con la caracterización que de este funcionario se les solicita, pues respondiendo al perfil recomendado por J. Brown y la NASP (entre otros), logran establecer como relevantes la empatía, la capacidad de escucha, la ética, la capacidad intelecto-cognitiva, la moral, la afectividad y la solidaridad.

Finalmente, el 50\% señala haber cambiado su visión del psicólogo educativo en los últimos tiempos.

\section{Limitaciones}

Necesario es reconocer que tanto el ítem 5 como el ítem 7 (su contraparte) de esta escala, debieron "invalidarse" por no evidenciar en su comportamiento una tendencia clara. Esto ocurrió pues erróneamente se construyó como un ítem abierto, cuando debieron haberse ofrecido los rangos de porcentaje (\%) de horario esperables para cada área de atención del psicólogo educativo. Por no considerar beneficioso el tener que forzar la clasificación, se obvió la información, aún cuando se evidencia el factor "horario" como uno de gran relevancia para la revisión interna del Instituto Andrea Jiménez.
Situación similar ocurrió con el ítem 11 , mismo que merece una redacción cuidadosa para la generalización posterior de esta investigación, ya que fue "mal interpretado" por las docentes participantes, perdiéndose la intención de conocer el "alcance del servicio" al enfocarlo como una intervención personal que para ellas ofrecía el psicólogo escolar. Nuevamente, y pese a lo antes anotado, parece evidente la concepción del psicólogo educativo como un "consejero" difuso en su ámbito de atención.

\section{Sugerencias}

para investigaciones posteriores

Con el ánimo de generalizar (a nivel nacional) los resultados descritos en este estudio, se sugiere la posibilidad de replicar el estudio en las escuelas de Educación Especial del gran área metropolitana y aquellas otras escuelas de ese tipo con que se cuente en otras regiones del MEP, viéndose favorecido así el servicio de psicología con que cuenta la mayoría de ellas.

Además, se considera fundamental el recomendar al Colegio Profesional de Psicólogos de Costa Rica y al Ministerio de Educación Pública, específicamente a su departamento de Educación Especial, el fomentar la divulgación y capacitación relati- 
va al "Rol del Psicólogo Educativo", a través de talleres, desplegables, páginas webb, buzones telefónicos, comités de formación-capacitación interinstitucionales, otro tipo de material impreso, etc., a psicólogos, docentes y familiares, además del público en general... Tal y como se iniciará en la escuela-base de este estudio.

\section{BIBLIOGRAFÍA}

CORNEL, E. L. (1942) The work of the school psychologist. Albany: Division of Research, New York State Education Department. Best Practices in School Psychology, NASP, 1995.

FAGAN T. K., (1990) "Trends in the History of School Psychology in USA" (1995) Best Practices in School Psychology, cap. 5, III Edited by Thomas \& Grimes: The National Asociation of School Psychologist, NASP Publications Policy Handbook, USA.

FAGAN T. K., (1993) "Separate but equal: School psychology s search for organizational identity". Journal of School Psychology 31, pp. 3-90, USA.

Franca-TARrago, O. (1996) Ética para Psicólogos: Introducción a la Psicoética. Editorial Descleé de Brower, S.A. $2^{\text {a }}$ Edición, pp. 282-285.

Fowler, E, \& Harrison P.L., (1996) “Best Practices in continuing Professional Development for School Psychologists", Best Practices in School Psychology, cap. 7, III Edited by Thomas \& Grimes: The National Asociation of School Psychologist, NASP Publications Policy Handbook, USA.
Galloway, D. (1998) "Special Education in the UK: Educational Psychologists and the Effectiveness of Special Education". School Psychology, UK. Review, Vol. 27, pp. 77-83, USA.

Good T. L.\& Brophy J., (1999) Psicología Educativa Contemporánea, $5^{\text {a }}$ Edición, Mc Graw-Hill, México.

HALL, M. E., (1949) “Current employment requirements for school psychologists". American Psychologist 4, pp. 519-525, USA.

Henson K. T. \& Eller B.F., (1999) Psicología Educativa para la enseñanza eficaz, International Thomson Editores, S.A., México.

NASP, (2000) "Professional Conduct Manual: Principles for Professional Ehics", Guidelines for the Provision of School Psychological Services.

OKLAND T. \& Cunnigham J., (1989) "The Future of School Psychology: Conceptual models for Its Development and examples of their Applications", cap. 2 "Models for Understanding School Psychologys Development and Future Trends", pp. 34-53, School Psychology Quarterly, USA.

SANDOVAL, J. (1993) "The history of interventions in school psychology". Journal of School Psychology 31, pp. 195-217, USA.

SHEPPARD, J. \& Dawson, M. (1998) Educational and School Psychology in the UK and US: an Introduction". School Psychology Review, Vol. 27, pp. 7-13., USA.

SpRINTHALL, N.A. \& Sprinthall R.C., (1990) Educational Psychology: A developmental approach, Quinta Edition, MC GrawHill, Inc., USA.

ZIGMOND, N, (1996) "Inclusion in Pensylvannia: educational experiences of students with disabilities in one elementary school". Journal of Special Education 29, pp. 124-132., USA. 